\title{
Relación entre el apoyo social, la satisfacción de las necesidades psicológicas básicas y el bienestar en adultos mayores
}

\author{
Elvira Tijeras, Lorena González-García, y Silvia Postigo \\ Universidad Europea de Valencia (España)
}

\begin{abstract}
La teoría de las necesidades psicológicas básicas postula que los contextos sociales en los que se promueve la satisfacción de las necesidades psicológicas básicas de autonomía, competencia y relación tienen un efecto positivo sobre el bienestar de las personas. En base a este marco teórico, el objetivo del presente trabajo fue analizar las posibles relaciones entre el apoyo social percibido por los mayores, la satisfacción de sus necesidades psicológicas básicas y su bienestar, medido a través de los indicadores de satisfacción con la vida y autoestima. Participaron 137 personas mayores. Los resultados mostraron que tanto el apoyo social percibido como la satisfacción de sus necesidades psicológicas básicas actúan como predictores positivos y significativos de su nivel de bienestar. Asimismo, los resultados revelaron que la satisfacción de las necesidades psicológicas básicas ejerce un papel mediador en la relación entre el apoyo social percibido y la satisfacción con la vida, aunque este papel no pudo ser demostrado en la relación entre el apoyo social y la autoestima. Los resultados enfatizan la importancia de desarrollar programas para promover un adecuado apoyo social y la satisfacción de las necesidades psicológicas básicas de los mayores con el objetivo de promover su bienestar.
\end{abstract}

Palabras clave: Adultos mayores, apoyo social percibido, necesidades psicológicas básicas, bienestar.

The relationship between social support, basic psychological needs satisfaction and well-being in elderly adults. Basic psychological needs theory defends that social contexts which promote basic psychological needs for autonomy, competence and relatedness, have a positive effect on people's well-being. Based on this theoretical framework, the aim of this study was to analyze the possible relationships between older people's perceived social support, their basic psychological needs satisfaction and their well-being, measured by life satisfaction and selfesteem. Participants of the study were 137 older people. Results of the study showed that both perceived social support and basic psychological needs satisfaction act as positive and significant predictors of elderly people's well-being. Finally, results revealed that basic psychological needs satisfaction functions as a mediator in the relationship between perceived social support and older people's life satisfaction. This mediational role couldn't be demonstrated in the relationship between perceived social support and self-esteem. These results emphasize the importance of developing programs that promote an adequate social support and the basic psychological needs satisfaction of the elderly people to promote their well-being.

Keywords: Elderly, perceived social support, basic psychological needs, well-being.

Correspondencia: Elvira Tijeras Salmerón. Departamento de Psicología. Paseo de la Alameda, 7. C.P.: 46010. Universidad Europea de Valencia, Valencia (España). E-mail: elviratijeras29@gmail.com 
La calidad de vida de las personas mayores es objeto de interés creciente en nuestra sociedad, pues constituyen el $9 \%$ de la población mundial, según los datos ofrecidos por el Banco Mundial (2017). Dado que este porcentaje sigue creciendo, es de especial relevancia desarrollar estrategias sociosanitarias adecuadas, que faciliten una mejor experiencia vital a este grupo poblacional y que nos ayuden a optimizar los recursos disponibles. Generalmente, la vejez se ha percibido como una etapa en la que se presentan múltiples pérdidas, entre las que destacan la pérdida de la condición física y/o cognitiva, la pérdida de familiares y amigos, así como la pérdida de roles (Cruz-Jentoft et al., 2010).

Resulta imposible evitar gran parte de estas pérdidas; sin embargo, es posible que la persona mayor se vincule en determinados tipos de actividades que favorezcan el mantenimiento óptimo de sus condiciones físicas, emocionales y sociales. En los últimos años, la investigación ha cambiado su visión de la vejez centrada en la pérdida y el deterioro por una visión que enfatiza aspectos positivos como disponer de tiempo libre para realizarse personalmente, tener la posibilidad de transmitir sus valores y experiencias a los más jóvenes, o llevar a cabo tareas como el cuidado de la familia (Arias e Iacub, 2013; Charles y Carstensen, 2007).

Una de las claves para el envejecimiento saludable es mantenerse activo física, mental y socialmente. Por ejemplo, la motivación por ser capaces de superar nuevos retos y esforzarse para conservar su contacto con otras personas y no perder sus redes de apoyo social se ha evidenciado como uno de los determinantes de la calidad de vida y la satisfacción vital de los adultos mayores (Farré, 1991).

Concretamente, el apoyo social percibido, entendido como la evaluación subjetiva del individuo sobre la adecuación del apoyo recibido o la apreciación del sujeto respecto de la calidad de sus relaciones significativas y del apoyo del que dispone (Barra, 2004), es uno de los factores clave para el bienestar global y sus dimensiones relacionadas (relaciones interpersonales positivas, autoaceptación y propósito en la vida), así como con la autoestima y la percepción de estabilidad (Lemos y Fernández, 1990; Vivaldi y Barra, 2012).

La teoría de las necesidades psicológicas básicas (BPNT, Basic Psychological Needs Theory) (Deci y Ryan, 2000; Ryan y Deci, 2017) es una teoría directamente centrada en el bienestar y el desarrollo óptimo de las personas, que considera el importante papel que el apoyo social juega sobre la satisfacción o frustración de las necesidades psicológicas básicas de las personas y sobre su bienestar.

Esta teoría postula la existencia de tres necesidades psicológicas básicas inherentes al ser humano, universales y definidas como nutrientes esenciales para el crecimiento psicológico, la integridad y el bienestar de las personas (Deci y Ryan, 2000). Estas necesidades son: 1) la necesidad de autonomía, entendida como el sentimiento de ser capaz de tomar decisiones sobre su propio comportamiento, regulando y siendo 
responsable de sus propias actitudes y siendo el origen de las mismas (DeCharms, 1968); 2) la necesidad de competencia, que expresa el esfuerzo de las personas por sentirse eficaces y hábiles en las tareas que llevan a cabo, poniendo en práctica sus capacidades (White, 1959); y 3) la necesidad de relación, referida a sentirse apreciado y cuidado por los demás, y sentir que cuida e íntima con los demás (Baumeister y Leary, 1995).

Estudios basados en la BPNT han evidenciado la importancia de cada una de estas tres necesidades para el bienestar (Deci y Ryan, 2000) y han demostrado que los contextos sociales que apoyan la autonomía de las personas favorecen la satisfacción de sus necesidades psicológicas básicas, lo que a su vez promueve un mayor bienestar y un menor malestar (Adie, Duda, y Ntoumanis, 2012; Baard, Deci, y Ryan, 2004; Kasser y Ryan, 2001). No obstante, este tipo de relaciones no se han estudiado aún en población de adultos mayores.

Por todo ello, en línea con el interés de promover la calidad de vida en las personas mayores y teniendo en cuenta las investigaciones previas en esta área de conocimiento, el objetivo general del presente estudio es analizar la relación entre el apoyo social percibido por los mayores, la satisfacción de sus necesidades psicológicas básicas y su bienestar. Para ello, se han delimitado los siguientes objetivos específicos: 1) analizar la relación entre el apoyo social percibido por los mayores, su percepción de satisfacción de las necesidades psicológicas básicas y su bienestar; y 2) analizar el papel mediador de las experiencias de satisfacción de las necesidades psicológicas básicas en la relación entre el apoyo social y el bienestar de los mayores.

En base a la literatura previa planteamos las siguientes hipótesis:

Hipótesis 1: El apoyo social, la satisfacción de las necesidades psicológicas básicas y los indicadores de bienestar se relacionarán de manera positiva y significativa.

Hipótesis 2: El apoyo social y la satisfacción de las necesidades psicológicas básicas predecirán positiva y significativamente la satisfacción con la vida y la autoestima de los participantes.

Hipótesis 3: La satisfacción de las necesidades psicológicas básicas actuará como mediador en la relación entre el apoyo social percibido por los mayores y los indicadores de bienestar empleados en el estudio.

\section{MÉTODO}

\section{Participantes}

Los participantes son 137 personas $\left(n_{\text {mujeres }}=106 ; 77.37 \%\right)$ con edades comprendidas entre 61 y 91 años $\left(M_{\text {edad }}=73.11 ; D T=6.22\right)$, residentes en 11 localidades de la Comunidad Valenciana. Respecto a su lugar de origen, 135 habían nacido en España (98.5 \%), 1 en Italia (0.7\%) y 1 en Estados Unidos (0.7\%). Atendiendo 
al estado civil, 78 estaban casados (58.6\%), 48 viudos (36.1\%) y 7 solteros $(5.3 \%)$, cuatro participantes no contestaron a este ítem.

El número de personas que conviven en el hogar de los participantes oscilaba entre 1 y 6 personas $(M=1.92 ; D T=0.81)$. Por último, el $49.6 \%$ de los participantes tenía un rango de ingresos total en el hogar entre 501-1000 €, seguido del $24.1 \%$ que ingresaba entre $1001-2000 €$, el $13.1 \%$ que ingresaba más de $2000 €$ y el $7.3 \%$ que ingresaba menos de $500 €$.

\section{Instrumentos}

Datos sociodemográficos. Se elaboraron 9 preguntas ad hoc para obtener información sobre la edad, sexo, localidad de residencia, país de nacimiento, estado civil, personas que conviven en el hogar y rango de ingresos salariales en el hogar.

Apoyo social percibido. Se utilizó la versión española (Bellón, Delgado, y Lardelli, 1996) del Cuestionario Duke-UNC-11 (Broadhead, Gehlbach, Degruy, y Kaplan, 1988), compuesto por 11 ítems con respuesta escalar tipo Likert de 5 puntos (1 "mucho menos de lo que desea"; 5 "tanto como desea"). El cuestionario permite agrupar las puntaciones en dos escalas: 1) la subescala de apoyo confidencial, compuesta por 7 ítems como "Tengo la posibilidad de hablar con alguien de mis problemas en el trabajo o en casa"; y 2) la subescala afectiva, que consta de 4 ítems como "Cuento con personas que se preocupan por lo que me sucede". La fiabilidad y la validez de este cuestionario ha sido observada en distintos estudios tanto en población general como en adultos mayores y personas cuidadoras (Ayala et al., 2012; Broadhead et al., 1988; Cuéllar-Flores y Dresch, 2012).

Satisfacción de las necesidades psicológicas básicas. Se utilizó la Escala de Satisfacción de las Necesidades Psicológicas Básicas (Deci y Ryan, 2000; Gagné, 2003) en su versión española (García-Merita, Castillo, Valcárcel, Pons, y Balaguer, 2005), formada por 11 ítems con una escala de respuesta tipo Likert de 7 puntos que van desde 1 "totalmente en desacuerdo" hasta 7 "totalmente de acuerdo", agrupados en tres dimensiones: 1) la satisfacción de la necesidad de autonomía se evalúa a través de ítems como "Siento como si fuera libre para decidir por mí mismo/a como quiero vivir mi vida"; 2) la satisfacción de la necesidad de competencia se evalúa con ítems como: "Recientemente he sido capaz de aprender nuevas e interesantes habilidades"; y 3 ) la dimensión de satisfacción de la necesidad de relación incluye afirmaciones como "Me gusta realmente la gente con la que me relaciono". Este instrumento ha demostrado adecuadas propiedades psicométricas en su versión española (García-Merita et al., 2005).

Satisfacción con la vida. Se empleó la Escala de Satisfacción con la Vida (Diener, Emmons, Larsen, y Griffin, 1985) en su versión española (Atienza, Pons, Balaguer, y García-Merita, 2000), compuesta por 5 ítems como "Hasta ahora he 
conseguido de la vida las cosas que considero importantes", con escala de respuesta tipo Likert de 7 puntos, donde 1 es "muy en desacuerdo" y 7 es "muy de acuerdo". La validez y fiabilidad de esta escala ha sido observada en estudios con población adulta (Moyano, Martínez, y Muñoz, 2013).

Autoestima. El instrumento utilizado para evaluar la autoestima global percibida por los encuestados fue la Escala de Autoestima de Rosenberg (Rosenberg, 1965) en su versión española (Atienza, Moreno, y Balaguer, 2000). Esta escala está compuesta por 10 ítems con escala de respuesta tipo Likert de 4 puntos, siendo 1 "muy en desacuerdo" y 4 "muy de acuerdo". Cinco de los ítems son afirmaciones positivas como "Soy capaz de hacer las cosas tan bien como los demás" y 5 son ítems inversos como "Me gustaría tener más respeto por mí mismo". La fiabilidad y validez de la escala ha sido evidenciada previamente en población de adultos mayores (De León y García, 2016).

\section{Procedimiento}

El estudio fue aprobado por el comité ético de la Universidad Europea. Una vez obtenida dicha aprobación, se contactó con ayuntamientos y asociaciones de jubilados de diferentes municipios cercanos a Valencia para informar sobre el proyecto y solicitar su participación. Cada ayuntamiento y/o asociación se encargó de citar a los encuestados que cumplieran con los requisitos previamente acordados: 1) tener más de 60 años; 2) vivir de manera autónoma (sin condiciones de dependencia); y 3) no padecer ninguna enfermedad incapacitante.

La recogida de información se realizó entre octubre y diciembre de 2018. Previamente, se recogió la firma del consentimiento informado para participar en la investigación, detallando su carácter voluntario y anónimo, y que podían abandonar la investigación en cualquier momento si así lo deseaban. Asimismo, se explicó la importancia de que contestaran a todas las preguntas. Durante todo el proceso de cumplimentación de los cuestionarios, los participantes disponían de ayuda de los miembros del equipo de investigación. El tiempo estimado para completar el cuestionario fue de 30 minutos.

\section{Análisis de datos}

Para alcanzar los objetivos planteados en el estudio se analizó, en primer lugar, la fiabilidad de los instrumentos de medida utilizados en el estudio, mediante el coeficiente Alfa de Cronbach. Asimismo, se llevaron a cabo análisis descriptivos de las variables sociodemográficas, correlaciones bivariadas (coeficiente de Pearson) para analizar la relación entre las variables de estudio, regresiones lineales para la predicción de las variables dependientes (satisfacción con la vida y autoestima) a través de las variables independientes (apoyo social y satisfacción de las necesidades psicológicas 
básicas), y regresiones jerárquicas para estudiar la mediación de la satisfacción de las necesidades psicológicas básicas. Todos los análisis se realizaron mediante el software estadístico SPSS Statistics V.24.

\section{RESULTADOS}

Los resultados muestran índices de fiabilidad adecuados en todas las escalas (Tabla 1), oscilando entre $\alpha=.67$ y $\alpha=.84$. En la tabla 1 se presentan también los estadísticos descriptivos de las variables de estudio. Los participantes presentan valores por encima de la media del cuestionario en apoyo social, así como en las subescalas de apoyo social confidencial y apoyo social afectivo. En relación con la satisfacción de las necesidades psicológicas básicas, observamos que los participantes presentan puntuaciones superiores a la media en la satisfacción de las tres necesidades en su conjunto.

Al analizar los resultados por separado, observamos que la necesidad que obtiene puntuaciones medias más elevadas es la necesidad de relación, seguida de la autonomía y finalmente la competencia. En cuanto a los indicadores de bienestar, los participantes presentan puntuaciones por encima de los valores medios del cuestionario en satisfacción con la vida y en la autoestima.

Tabla 1. Estadísticos descriptivos de las variables utilizadas en el estudio

\begin{tabular}{lccccc}
\hline & $N$ & Rango respuesta & $M$ & $D T$ & Alfa de Cronbach \\
\hline Apoyo Social & 137 & $1-5$ & 3.93 & 0.74 & .82 \\
\hline AS Confidencial & 137 & $1-5$ & 3.78 & 0.82 & .78 \\
\hline AS Afectivo & 137 & $1-5$ & 4.20 & 0.79 & .70 \\
\hline NPB & 137 & $1-7$ & 5.97 & 0.89 & .84 \\
\hline NPB Autonomía & 137 & $1-7$ & 6.09 & 0.95 & .68 \\
\hline NPB Competencia & 137 & $1-7$ & 5.58 & 1.21 & .67 \\
\hline NPB Relación & 137 & $1-7$ & 6.15 & 0.94 & .77 \\
\hline Satisfacción Vida & 136 & $1-4$ & 4.99 & 1.27 & .81 \\
\hline Autoestima & 137 & $1-4$ & 3.14 & 0.50 & .71 \\
\hline
\end{tabular}

Los análisis correlacionales (Tabla 2) indican que el apoyo social percibido se relaciona de forma significativa y positiva con la satisfacción de las tres necesidades psicológicas básicas, evaluadas en conjunto $(r=.54 ; p<.05)$ y de forma separada: autonomía $(r=.52 ; p<.001)$, competencia $(r=.48 ; p<.001)$ y relación $(r=.41$; $p<.001)$.

Asimismo, las sub-dimensiones del apoyo social (confidencial y afectivo) también se relacionan positiva y significativamente con las tres necesidades psicológicas básicas evaluadas en su conjunto, así como con cada una de las necesidades psicológicas básicas. La relación entre el apoyo social y los indicadores de bienestar también es positiva, tanto con la satisfacción con la vida $(r=.34 ; p<.001)$ como con la autoestima $(r=.21 ; p<.05)$. 
Las necesidades psicológicas básicas evaluadas en conjunto se relacionan significativa y positivamente con la satisfacción con la vida $(r=.43 ; p<.05)$ y con la autoestima $(r=.19 ; p<.05)$. Una vez desglosadas las tres necesidades psicológicas básicas, analizamos su relación con la satisfacción con la vida y la autoestima, observando que las únicas variables que no guardan una relación significativa entre sí son la satisfacción de la necesidad psicológica básica de relación y la autoestima $(r=.011 ; p>.05)$.

Tabla 2. Correlaciones entre las variables de estudio

\begin{tabular}{|c|c|c|c|c|c|c|c|c|}
\hline & 1 & 2 & 3 & 4 & 5 & 6 & 7 & 8 \\
\hline \multicolumn{9}{|l|}{ 1.AS } \\
\hline 2.AS Confidencial & $.95 * * *$ & & & & & & & \\
\hline 3.AS Afectivo & $.83 * * *$ & $.62 * * *$ & & & & & & \\
\hline 4.NPB & $.54 * * *$ & 47 *** & $.54 * * *$ & & & & & \\
\hline 5.NPB Autonomía & $.52 * * *$ & $46^{* * *}$ & $.49 * * *$ & $.93 * * *$ & & & & \\
\hline 6.NPB Competencia & $.48 * * *$ & $.42 * * *$ & $.46^{* * *}$ & $.86 * * *$ & $68 * * *$ & & & \\
\hline 7.NPB Relación & $.41 * * *$ & $.33 * * *$ & $.47 * * *$ & $.82 * * *$ & $.70 * * *$ & .56 *** & & \\
\hline 8.Satisfacción Vida & $.34 * * *$ & $.34 * * *$ & $.24 * *$ & $.43 * * *$ & $.36 * * *$ & $.45 * * *$ & $.31 * * *$ & \\
\hline 9.Autoestima & $.21 *$ & $.23 * *$ & .11 & $.19 *$ & $.24 * *$ & $.22 *$ & .01 & $.19^{*}$ \\
\hline
\end{tabular}

En cuanto al análisis de regresiones lineales, los resultados indican que el apoyo social predice significativa y positivamente la satisfacción con la vida $(F=17.363 ; g l=1 ; p<.001)$, explicando el $11.5 \%$ de la varianza. Mientras que la satisfacción de las necesidades psicológicas básicas predice significativa y positivamente la satisfacción con la vida $(F=30.141 ; g l=1 ; p<.001)$, explicando el $18.4 \%$ de la varianza.

Respecto a los análisis de mediación, se observa que, analizando el efecto de las dos variables independientes (apoyo social percibido y satisfacción de las necesidades psicológicas básicas) sobre la variable dependiente de satisfacción con la vida y el posible efecto mediador de la satisfacción de las necesidades psicológicas básicas, los resultados confirman que el apoyo social predice positiva y significativamente $(F=17.363 ; g l=1 ; p<.001)$ la satisfacción con la vida. Sin embargo, al introducir las dos variables independientes en el análisis de regresión jerárquica, el apoyo social deja de predecir significativamente la satisfacción con la vida, mientras que la satisfacción de las necesidades psicológicas básicas sí que la predice positiva y significativa $(F=16.880 ; g l=2 ; p<.001)$. Esto sugiere que la satisfacción de las necesidades psicológicas básicas media de forma total la relación entre el apoyo social y la satisfacción con la vida de los participantes. Las dos variables independientes en conjunto (apoyo social y satisfacción de necesidades psicológicas básicas) explican el $20.2 \%$ de la varianza de la satisfacción con la vida de los mayores.

En cuanto a la predicción de la autoestima como indicador de bienestar, los resultados indican que el apoyo social predice significativa y positivamente la 
autoestima $(F=5.953 ; g l=1 ; p<.05)$. Concretamente, el apoyo social explica el $4.2 \%$ de la varianza de la autoestima de las personas mayores. Asimismo, los resultados muestran que la satisfacción de las necesidades psicológicas básicas predice significativa y positivamente la autoestima $(F=5.282 ; g l=1 ; p<0.05)$, explicando el $3.8 \%$ de la varianza. Al analizar el efecto de las dos variables independientes sobre la autoestima y el posible efecto mediador de la satisfacción de las necesidades psicológicas básicas, los resultados confirman que el apoyo social predice positiva y significativamente $(F=5.953 ; g l=1 ; p<.05)$ la satisfacción con la vida. Sin embargo, cuando se introducen simultáneamente las dos variables independientes en el análisis de regresión jerárquica, tanto el apoyo social como la satisfacción de las necesidades psicológicas básicas dejan de predecir significativamente la autoestima $(F=3.672 ; g l=2 ; p<.05)$.

\section{DISCUSIÓN Y CONCLUSIONES}

A pesar de que, durante muchos años, gran parte de la investigación sobre la vejez se ha limitado a resaltar los aspectos negativos de esta etapa de la vida como el desarrollo de enfermedades, el deterioro cognitivo o la necesidad de llevar una vida sosegada (Arias e Iacub, 2013), deben tenerse en cuenta los matices positivos que conlleva, destacando la capacidad de los adultos mayores para fortalecer el entorno familiar, comunicar experiencias a otros y autorrealizarse (Sanhueza et al., 2005).

Al igual que estudios previos (Zavala et al., 2006), los resultados de este trabajo muestran que los adultos mayores obtienen puntuaciones superiores a la media en los indicadores de bienestar (satisfacción con la vida y autoestima), satisfacción de las necesidades psicológicas básicas y apoyo social percibido. Estos resultados señalan que las personas mayores pueden sentirse competentes en lo que hacen, con autonomía para tomar sus propias decisiones y con buena relación con las personas que les importan, además de experimentar bienestar y apoyo social, sugiriendo la importancia de no generalizar los factores desfavorables de la vejez y orientar el estudio hacia las diferencias individuales.

Los resultados muestran evidencias positivas sobre la primera y la segunda hipótesis, mostrando que el apoyo social percibido y la satisfacción de las necesidades psicológicas básicas se relacionan significativa y positivamente con el bienestar de los adultos mayores, medido a través de los indicadores de satisfacción con la vida y autoestima. Además, los resultados muestran que tanto el apoyo social como las necesidades predicen ambos indicadores de bienestar. Estos resultados son congruentes con estudios anteriores que señalan que ambas variables tienen beneficios sobre la salud de las personas mayores como el aumento de la autoestima (Vivaldi y Barra, 2012), de la satisfacción con la vida (San Martín y Barra, 2013), y el sentimiento de felicidad (Kasser y Ryan, 2001). 
Tomando en consideración estos beneficios, es importante que el contexto del adulto mayor facilite tener una red de apoyo social diaria, así como incluir los factores de apoyo social y satisfacción de las necesidades psicológicas básicas en la elaboración de estrategias y programas de intervención dirigidos a mejorar la calidad de vida de las personas mayores.

Las hipótesis sobre el papel mediador de la satisfacción de las necesidades psicológicas básicas en la relación entre el apoyo social y el bienestar de las personas mayores han sido parcialmente confirmadas. Los resultados muestran un efecto de mediación total de las necesidades entre el apoyo social y la satisfacción con la vida. Sin embargo, al examinar el papel mediador de las necesidades psicológicas básicas en la relación entre el apoyo social percibido y la autoestima de los participantes, observamos que no existe mediación por parte de dicha variable.

Los resultados obtenidos en estudios previos demuestran que los adultos mayores institucionalizados que tenían relaciones con otros y percibían que se apoyaba su autonomía tenían un mayor bienestar y felicidad (Kasser y Ryan, 2001). Asimismo, el papel mediador de la satisfacción de las necesidades psicológicas básicas en la relación entre apoyo social percibido y el bienestar de las personas ha sido corroborado en otras poblaciones (Adie et al., 2012).

Los resultados del presente estudio señalan la necesidad de favorecer aspectos que satisfagan las necesidades psicológicas básicas de los adultos mayores, puesto que estas no sólo tienen un papel importante en su calidad de vida, sino que también actúan como mediadoras entre el apoyo social percibido y su bienestar; por ejemplo, cuando se perciben a sí mismas como personas autónomas y funcionales, con capacidad para realizar distintas tareas. En conclusión, aunque la percepción de apoyo social se relaciona positiva y significativamente con la satisfacción con la vida, es la satisfacción de las necesidades básicas la que ejerce un papel indispensable en esta relación. No obstante, los resultados de este estudio no permiten alcanzar la misma conclusión en cuanto al papel mediador de las necesidades en la relación entre el apoyo social y la autoestima; por lo que sería interesante realizar nuevas investigaciones para contrastar los resultados obtenidos.

Estos resultados pueden ser relevantes a la hora de desarrollar intervenciones dirigidas a aumentar el bienestar y la calidad de vida de los adultos mayores, pues, además de considerar los efectos beneficiosos del apoyo social percibido, es indispensable tener en consideración el papel mediador que desempeñan las necesidades psicológicas básicas. Esto implica que, con el objetivo de fomentar el bienestar de las personas mayores, los programas deben considerar el favorecer contextos en los que las personas mayores puedan percibir un buen ambiente social, en el que se faciliten los sentimientos de autonomía, competencia y relación con otros, para que sientan que son capaces de decidir por sí mismos y se sientan competentes en las actividades que llevan 
a cabo, que son valorados por los demás y que realizan una aportación a su vida y a la de otros.

\section{REFERENCIAS}

Adie, J.W., Duda, J.L., y Ntoumanis, N. (2012). Perceived autonomy support, basic need satisfaction and the well- and ill-being of elite youth soccer players: A longitudinal investigation. Psychology of Sport and Exercise, 13, 51-59.

Arias, C.J., e Iacub, R. (2013). ¿Por qué investigar aspectos positivos en la vejez? Contribuciones para un cambio de paradigma. Ciencias Humanas, Lingüística, Letras y Artes, 21(2), 271-281.

Atienza, F., Pons, D., Balaguer, I., y García-Merita, M. (2000). Propiedades psicométricas de la Escala de Satisfacción con la Vida en adolescentes. Psicothema, 12(1984), 314-319.

Atienza, F.L., Moreno, Y., y Balaguer, I. (2000). Análisis de la dimensionalidad de a escala de autoestima de Rosenberg en una muestra de adolescentes valencianos. Universitas Tarraconenses, 22, 29-42.

Ayala, A., Rodríguez-Blázquez, C., Frades-payo, B., João, M., Martínez-Martín, P., FernándezMayoralas, G., y Rojo-Pérez, F. (2012). Propiedades psicométricas del Cuestionario de Apoyo Social Funcional y de la Escala de Soledad en adultos mayores no institucionalizados en España. Gaceta Sanitaria, 26(4), 317-324. doi:10.1016/j.gaceta.2011.08.009

Baard, P.P., Deci, E.L., y Ryan, R.M. (2004). Intrinsic need satisfaction: A motivational basis of performance and weil-being in two work settings. Journal of Applied Social Psychology, 34(10), 2045-2068.

Banco Mundial (2017). Población de 65 años y más. Recuperado de: https://datos.bancomundial.org/indicador/sp.pop.65up.to.zs

Barra, E. (2004). Apoyo social, estrés y salud. Psicología y Salud, 14(2), 237-243.

Baumeister, R.F., y Leary, M.R. (1995). The need to belong: Desire for interpersonal attachments as a fundamental human motivation. Psychological Bulletin, 117(3), 497-529.

Bellón, J.A., Delgado, A., y Lardelli, P. (1996). Validación del cuestionario de Apoyo Social Funcional Duke-UNK-11 en personas cuidadoras. Atención Primaria, 18, 153-163.

Broadhead, W.E., Gehlbach, S.H., Degruy, F.V., y Kaplan, B.H. (1988). The Duke-UNC Functional Social Support Questionnaire: Measurement for social support in family medicine patients. Medicine Care, 26, 709-723.

Charles, S.T., y Carstensen, L.L. (2007). Emotion Regulation and Aging. En J. J. Gross (Eds.), Handbook of emotion regulation (pp. 307-327). New York: The Guilford Press.

Cruz-Jentoft, A.J., Baeyens, J.P., Bauer, J.M., Boirie, Y., Cederholm, T., Landi, F., ... Zamboni, M. (2010). Sarcopenia: European consensus on definition and diagnosis. Age and Ageing, 39(4), 412-423. doi:10.1093/ageing/afq034

Cuéllar-Flores, I., y Dresch, V. (2012). Validación del cuestionario de Apoyo Social Funcional Duke-UNK-11 en personas cuidadoras. Revista Iberoamericana de Diagnóstico y Evaluación, 2(34), 89-101.

De León, C.A., y García, M. (2016). Escala de Rosenberg en población de adultos mayores. Ciencias Psicológicas, 10(2), 119-127.

Decharms, R. (1968). Personal causation. New York: Academic Press.

Deci, E.L., y Ryan, R.M. (2000). The "what" and "why" of Goal Pursuits: of Behavior Human Needs and the Self-determination. Psychological Inquiry, 11(4), 227-268. doi:10.1207/S15327965PLI1104 
Diener, E., Emmons, R.A., Larsen, R.J., y Griffin, S.L.A.E. (1985). The Satisfaction with Life Scale. Journal of Personality Assessment, 49(1), 71-75. doi:10.1207/s15327752jpa4901_13

Farré, A.F. (1991). Autopercepción del proceso de envejecimiento en la mujer entre 50 y 60 años. Anuario de Psicología, 50, 67-78.

Gagné, M. (2003). The role of autonomy support and autonomy orientation in prosocial behavior engagement. Motivation and Emotion, 27, 199-223.

García-Merita, M., Castillo, I., Valcárcel, P., Pons, D., y Balaguer, I. (2005). Psychometric properties of the Basic Need Satisfaction in Life Scale in a Spanish version. In 9th European Congress of Psychology. Granada, España.

Kasser, T., y Ryan, R.M. (2001). Be careful what you wish for: Optimal functioning and the relative attainment of intrinsic and extrinsic goals. Life Goals and Well-Being: Towards a Positive Psychology of Human Striving, 1, 116-131.

Lemos, S., y Fernández, J.R. (1990). Redes de soporte social y salud. Psicothema, 2(2), 113-135.

Moyano, N.C., Martínez, M., y Muñoz, M.P. (2013). Propiedades psicométricas de la Escala de Satisfacción con la Vida de Diener. Revista Argentina de Clínica Psicológica, 22(2), 161-168.

Rosenberg, M. (1965). Society and the adolescent self-image. Princeton, NJ: Princeton University Press.

Ryan, R.M., y Deci, E.L. (2017). Self-determination theory: Basic psychological needs in motivation, development, and wellness. New York: Guilford Publications.

San Martín, J.L., y Barra, E. (2013). Autoestima, apoyo social y satisfacción vital en adolescentes. Terapia Psicológica, 31(3), 287-291.

Sanhueza, M., Castro, M., y Merino, J.M. (2005). Adultos mayores funcionales: Un nuevo concepto en salud. Ciencia y Enfermería, 11(2), 17-21.

Vivaldi, F., y Barra, E. (2012). Bienestar Psicológico, Apoyo Social Percibido y Percepción de Salud en Adultos Mayores. Terapia Psicológica, 30(2), 23-29.

White, R.W. (1959). Motivation reconsidered. Psychological Review, 66, 297-333.

Zavala, M., Vidal, D., Castro, M., Quiroga, P., y Klassen, G. (2006). Funcionamiento social del adulto mayor. Ciencia y Enfermería, 12(2), 53-62.

Recibido: 25 de septiembre de 2019

Recepción Modificaciones: 20 de octubre de 2019

Aceptado: 23 de octubre de 2019 